
( 1 3 1 2 3 4 5 6

\title{
The value of wastewater-based epidemiology in the estimation of alcohol consumption
}

Ester López-García ${ }^{1}$, Cristina Postigo ${ }^{1}$, Damià Barceló ${ }^{1,2}$, Miren López de Alda ${ }^{1}$

${ }^{1}$ Water, Environmental and Food Chemistry (ENFOCHEM), Department of Environmental Chemistry, Institute of Environmental Assessment and Water Research (IDAEA-CSIC), Jordi Girona 18-26, 08034, Barcelona, Spain.

${ }^{2}$ Catalan Institute for Water Research, H2O Building, Scientific and Technological Park of the University of Girona, Emili Grahit 101, 17003, Girona, Spain 10 12

*Corresponding Authors: Cristina Postigo, Miren López de Alda Institute of Environmental Assessment and Water Research (IDAEA-CSIC), Department of Environmental Chemistry C/ Jordi Girona 18-26, 08034 Barcelona, Spain. cprqam@cid.csic.es, mlaqam@cid.csic.es Tel: +34-934-006-100, Fax: +34-932-045-904 


\section{Highlights}

28 - Ethyl sulfate is measured in wastewater to back-calculate alcohol consumption

- WBE-data (local) are usually lower than WHO country-based data

- Main uncertainties are related to population-size estimates and ethanol metabolism

- Valid tool to rapidly derive changes in alcohol consumption patterns

Abstract

37 The present review discusses the value of wastewater-based epidemiology (WBE) to 38 estimate alcohol consumption in a given population. It gives an overview of the works 39 conducted in this respect to date, and the comparability of the WBE-derived data with 40 official estimates provided by the World Health Organization, generated after survey and 41 alcohol sales data. Finally, it provides insights to the main advantages and limitations of the 42 WBE approach over traditional methods, and aspects/knowledge to be 43 improved/investigated.

46 Keywords: sewage epidemiology, ethyl sulfate (EtS), alcohol consumption, ethanol 47 metabolism 


\section{1. Introduction}

52 Alcohol is one of the most used recreational substances in the world. According to the

53 World Health Organization (WHO), almost half of the worldwide population (43\%)

54 consumed alcohol in the last 12 months in 2016. Alcohol consumption increased globally

55 between 2005 and 2010 from 5.5 L to 6.4 L per capita (aged over 15 years ) and has

56 remained stable thereafter until 2016 [1]. Excessive alcohol consumption has been linked to

57 diverse social problems (suicides, homicides, robberies, violence, traffic accidents, etc.) and

58 health problems (digestive, infectious and cardiovascular diseases, cancer, etc.). Harmful

59 use of alcohol was the cause of $5.3 \%$ of all deaths worldwide in 2016 [1]. Thus, the

60 availability of tools to track alcohol consumption in time and evaluate the reliable

61 effectiveness of adopted measures to reduce its excessive use is important.

62 The prevalence of alcohol use is commonly estimated through population surveys [2, 3] and

63 sales statistics [4]. However, these methods are time-consuming and expensive. Moreover,

64 they do not always provide reliable data due to widespread under-reporting in surveys [5]

65 or unaccounted smuggled, stored and disposed alcohol in total sales volumes. Alternatively,

66 a novel approach known as wastewater-based epidemiology (WBE) has been applied in

67 recent years to assess alcohol use in a given population. WBE back-calculates alcohol

68 consumption from the concentration of an alcohol consumption indicator, most commonly

69 ethyl-sulfate (EtS), found in wastewater. Thus, WBE provides anonymous and objective

70 information in a fast and relatively economic way. However, this approach is also subject to

71 different sources of uncertainty. This review aims at (i) presenting the WBE approach to

72 estimate alcohol use by a specific population, (ii) discussing its utility based on the peer-

73 reviewed studies published so far on this topic, and (iii) highlighting its advantages and 74 limitations.

\section{Basis for estimation of alcohol use using WBE}

77 WBE relies on the fact that wastewater is a diluted pooled urine sample of a specific 78 population that can provide valuable information if appropriate biomarkers are monitored. 
After consumption of any alcoholic beverage, most of its ethanol content $(-95 \%)$ is metabolized in the liver via oxidation to acetaldehyde and acetic acid. However, a very small fraction $(<0.1 \%)$ is converted to ethyl glucuronide $(\mathrm{EtG})$ and $\mathrm{EtS}$ after conjugation with glucuronic acid and sulfate, respectively. These metabolites are excreted together with unaltered ethanol (5\%). EtG and $\mathrm{EtS}$ are both good indicators of recent alcohol consumption, because they are excreted within a few hours after ingestion and are detectable in urine for 1 or 2 days, depending on the subject and the alcohol dose [6]. However, only EtS has been used as a biomarker of alcohol consumption because it is more stable in wastewater than EtG. Lab-scale experiments showed that EtS concentration in wastewater remained stable for at least one week at room temperature and for more than one month at $-20^{\circ} \mathrm{C}$ [7]. On the contrary, EtG concentration decreased up to $50 \%$ after $18 \mathrm{~h}$ at room temperature [8]. In addition, EtS is not formed in wastewater through metabolization of unaltered ethanol by endogenous bacteria, and hence, its presence in wastewater can be only attributed to alcohol consumption [8]. This makes EtS an exclusive and unequivocal biomarker to be used in WBE for back-calculation of alcohol consumption according to Equation 1,

$\frac{m L \text { of EtOH }}{\text { day*person }}=C_{E t S}\left[\frac{\mu g}{L}\right] * 10^{-6}\left[\frac{g}{\mu g}\right] * Q\left[\frac{m^{3}}{\text { day }}\right] * 10^{3}\left[\frac{L}{m^{3}}\right] * \frac{1}{P(\text { persons })} * F * \frac{1}{\rho_{E t O H}\left[\frac{g}{m L}\right]}$

$F=\left[\frac{M_{E t o H} / M_{E t S}}{\% \text { excrection rate EtS }} * 100\right]$

where $\mathrm{C}_{\mathrm{EtS}}$ is the concentration of $\mathrm{EtS}$ in wastewater at the entrance of a wastewater treatment plant (WWTP), Q is the water flow entering the WWTP during the sampling event, $\mathrm{P}$ is the population connected to the WWTP, F is a correction factor that takes into account the molar mass ratio between ethanol and EtS (0.365) and the excretion rate of EtS (see Table 1 and Equation 2), and $\rho_{\text {EtOH }}$ is ethanol density $(0.789 \mathrm{~g} / \mathrm{mL})$.

Two approaches have been used to estimate alcohol consumption, providing fully comparable results [8]. One is the classical average mass-flow method that is ideal for 
compounds that have a long excretion half-life in urine or are consumed at a constant rate throughout the day and the night by many people in the studied area. The other one is based on the EtS excretion profile. This approach may provide more accurate consumption figures for compounds with short urinary excretion half-life, as it is the case for EtS (3 hours).

\section{Applicability and value of WBE to estimate alcohol consumption}

The first study that applied WBE to estimate alcohol use dates from 2011 [8]. Since then, various studies, summarized in Table 1, have been conducted in this line to explore i) the value of WBE to estimate alcohol consumption, and ii) the comparability of the data generated with this approach and traditional methods, i.e, official estimates provided by WHO and each country institutions. To date, WBE alcohol consumption figures have been derived from EtS loads entering 55 WWTPs spread in 13 different countries all over the world [8-19]. As shown in Table 1, most of the studies were conducted in major cities from different European countries.

Oslo was the first city where WBE was applied to estimate alcohol consumption. For this, samples were collected daily for 25 consecutive days at the entrance of one WWTP. In this study, two calculation methods to estimate alcohol use were evaluated and compared: the classical average mass-flow method and a novel method based on the EtS excretion profile. Equal alcohol consumption figures were obtained with both approaches $(4,900$ to 7,800 $\mathrm{Kg} / \mathrm{day})$. These data were in very good agreement with Norway sales statistics $(6,750$ $\mathrm{Kg} / \mathrm{day}$ ), which are among the most accurate in the world because of the strict control of import and sale of alcoholic beverages by the government [8]. In areas like the one investigated in this study, where alcohol is mostly consumed over a short period of time (usually during the evening), a limited number of samples can be used to derive alcohol use figures through the excretion profile integration method (morning peak of Et-S in wastewater associated with morning urination) [8].

Following this study, the average mass-flow method was applied to estimate alcohol use in Milan [17], the Spanish cities Santiago de Compostela [7] and Barcelona [14], and in multi- 
city studies conducted at international [18] and European level [10]. In all of these studies, annual consumptions were derived from one-week sampling at one WWTP, except in the case of Milan where samples were collected for two weeks. The highest consumption rates (above $25 \mathrm{~mL} /$ day/inhabitant) were obtained for cities in Canada (Granby and Montreal), Denmark (Copenhagen) and Germany (Munich and Dresden). City based-WBE values were in good agreement $(<15 \%)$ with country based-WHO data only in a few cases, i.e., Dresden, Munich, Castellón, Amsterdam, and Eindhoven. Mastroianni et al. [14] also reported a good agreement between wine consumption data derived from WBE and figures reported by the Spanish Ministry of Agriculture.

Overall, the low comparability of figures provided by WHO and WBE could be attributed to: i) the small geographic coverage of the WBE method (based on only one city or part of it) as compared to WHO data (based on nation-wide surveys and sales data), and to the fact that the area monitored by means of WBE may not be representative of country trends in alcohol consumption; ii) the short sampling periods used to derive WBE data (usually one "normal" week is selected to generate annual consumption data); iii) the uncertainty associated to the WBE methodology (see further discussion in section 5), and iv) imprecise estimates derived from surveys due to underreporting and sales data in areas where purchased alcohol may not be immediately consumed.

A couple of studies used EtS loads entering various WWTPs to derive national alcohol consumption figures. This was done by Boogaerts et al. in Belgium, [11], and by Lai et al. in Australia [13], who covered $12 \%$ and $45 \%$ of the country's total population, respectively. In the case of Belgium, the WBE-derived figure was below the one reported by WHO. However, it was in agreement with alcohol use figures provided by the Belgian Scientific Institute of Public Health. In the Australian study, average alcohol consumption figures obtained were very similar to those reported by the National Drug Strategy Household Survey (NDSHS). However, contrary to NDSHS data, WBE figures revealed large differences in alcohol consumption between rural and urban communities.

The comparability between alcohol consumption estimates produced by WBE and surveys in a medium-sized Belgian city was investigated by van Well et al. [19]. The study showed 
no correlation between WBE and survey data. However, authors attributed this to the small survey sample size (only $1 \%$ of survey response was obtained each study-week) [19].

Besides the comparison of WBE-data with survey data and official estimates, one of the main objectives of multi-city studies was investigating spatial differences in alcohol consumption. In addition to this, the collection of samples for seven consecutive days and the iteration of this exercise in consecutive years allowed investigating consumption patterns in time in each of the investigated cities throughout the week or throughout the years. As observed in the first study on this topic, conducted by Reid et al. [8], and several studies published afterwards, recreational use of alcohol resulted in higher EtS loads during the weekend than during weekdays $[9,11,14,15,17-19]$. In contrast, such profile was not observed in Lesvos Island (Greece), which could be attributed to the fact that alcohol drinking is a cultural aspect in Southern European countries [12].

The WBE approach also allows quantifying changes in alcohol consumption associated with special events, e.g., local festivities or festivals, when heavy drinking may take place. This was proved by Andrés-Costa et al. during the "Fallas" festivity in Valencia [9]. In this study, they found almost five and three times higher alcohol consumption during this festivity than during a normal week and weekend, respectively.

Recently, Chen et al. evaluated the WBE approach to estimate alcohol consumption in three different communities of the United States [20]. This study has been the longest conducted to date since samples were collected one weekday every month for 11 months. The average alcohol consumption obtained with the WBE approach was similar to those reported by the WHO and by the National Institute of Alcohol Abuse and Alcoholism (NIAAA). However, consumption figures were derived from samples collected on weekdays. In order to take into account weekend use, when alcohol use is presumably higher as compared to weekdays, they applied a correction factor (1.18) based on previously published studies. After correction, only one of the three investigated communities showed good agreement between the WHO and the WBE data. 


\section{Advantages of WBE for estimating alcohol consumption}

194 The main advantages that WBE presents over traditional methods to estimate alcohol use 195 are its capability of generating information on consumption habits in a fast, economic, 196 objective, and anonymous way.

197 Contrary to traditional methods, the WBE approach allows obtaining patterns of alcohol 198 consumption at a high spatial resolution. The coverage area of WBE-derived data is defined 199 by the wastewater catchment (i.e., population connected to the same sewage network) 200 where the sample is collected. Thus, consumption patterns in different size populations can 201 be well defined (see studies carried out in Australia [13], Belgium [11], and Greece[12]). 202 For instance, alcohol use in rural areas of Australia was three to four times higher than in 203 urban zones. On the contrary, alcohol consumption estimates for large cities in Belgium 204 and in Greece were higher than in small cities, although, in the case of Greece, differences 205 were not significant.

206 Due to the high-throughput character of the WBE approach, consumption trends in time can 207 be rapidly defined and changes in consumption patterns during special events can be 208 detected. For the same reason, it could be used to evaluate the effectiveness of preventive 209 measures (educational and sensibilization campaigns in schools or specific sub210 populations) or the efficacy of certain regulations.

\section{5. Limitations of WBE for estimating alcohol consumption}

213 The WBE approach to estimate alcohol use presents also some limitations and therefore it 214 has to be considered as a tool that complements traditional methods. The main limitation is 215 the uncertainty associated with this method that has to be considered when interpreting 216 WBE results. The sources of uncertainty of the WBE approach are well described [21]. 217 They are related to the sampling protocol, the analytical measurement, the stability of the 218 selected biomarker (EtS) in wastewater and in the sewer system, the metabolism of alcohol, 219 and the estimation of the population contributing to the EtS loads measured. The amount of 220 uncertainty associated with the WBE method is specific to each case study. This aspect has 
221 been poorly addressed in most of the studies conducted so far, and needs detailed

222 characterization and deeper understanding in future studies.

223 Uncertainty associated to wastewater sampling and EtS analysis can be minimized and 224 controlled if best practice protocols are followed (collection of 24-hour flow or time225 proportional integrated samples, sample preservation during collection and transport in cool conditions, use of deuterated surrogate standards for analysis, etc.) [21].

227 Regarding stability, EtS showed little or no significant degradation in wastewater at room temperature for 18h [8] and even one week [7] (in-sample stability). However, two recent 229 studies conducted in both real sewer [22] and lab-scale sewer reactors (rising main and 230 gravity reactors) that mimic in-sewer conditions [23] have shown that EtS is degraded to some extent in sewer systems (in-sewer stability). The study conducted in real sewer systems demonstrated that the degradation rate of EtS is approximately $8 \%$ per hour and this could vary from catchment to catchment [22]. The experiments done with lab-scale sewer reactors showed that degradation of EtS in rising main sewer reactors is higher than in gravity reactors and that $20 \%$ of EtS could degrade after 3 and 9 hours, respectively. These aspects have to be taken into consideration when estimating alcohol consumption by means of WBE, and also when comparing WBE-derived alcohol consumption data among populations, as they could lead to underestimating the real alcohol consumption.

239 Alcohol metabolism has been scarcely explored and needs further investigation to reduce the uncertainty associated. One of the studies conducted in this line showed that on a molar 241 basis, a median of $0.011 \%$ (range: 0.010-0.016) of the alcohol consumed was excreted as 242 EtS [24], while another study reported an EtS excretion rate of 0.022\% [25]. Both studies 243 were conducted using very few individuals (ten individuals in the first one and one 244 individual in the second one), and consequently, they may not be representative of the 245 general population, since age, gender, race, and health conditions could also affect alcohol metabolism. WBE alcohol consumption figures reported to date are based on different EtS 247 excretion rate values. As shown in Table 1, the median $0.011 \%$, the range $0.011-0.016 \%$, or 248 the people-weighted mean of the aforementioned studies $0.012 \%$ have been used for this 249 purpose. Based on this, ethanol metabolism needs to be further studied to define a single 250 and representative excretion rate of EtS. 
251 The size of the population that contributes to the Et-S loads measured in wastewater is an 252 important source of uncertainty. The method selected to estimate the population size 253 (census data, WWTP design capacity, biomarkers, or water quality parameters) was shown 254 in a multi-city study to affect illicit drug consumption rates to a different extent. Variations 255 as low as $7 \%$ or up to $55 \%$ were found in the different cities investigated [21]. Different 256 water quality parameters, such as biological oxygen demand, chemical oxygen demand, 257 total nitrogen and total phosphorus, and several human metabolites and pharmaceuticals 258 and personal care products have been proposed and investigated as potential markers that 259 can contribute to reduce uncertainty associated with the estimation of population size [21, 260 26, 27]. Ammonium $\left(\mathrm{NH}_{4}{ }^{+}\right)$, an indirect marker of urine that can be measured online in 261 wastewater, was reported to be an extremely useful marker to monitor fluctuations in 262 population size in WBE studies [28]. The use of mobile device data has been recently 263 proposed as a reliable tool to better estimate dynamic population size in a wastewater 264 catchment [29]. However, since this type of data may be not easily accessible or may be 265 costly, the most reliable estimation of population size has to be made in each case, after 266 consulting WWTP experts (origin of wastewater, municipalities connected to the WWTP, 267 and census data) and considering the population dynamics of each investigated area 268 (presence of tourists, commuters, holiday periods, etc.). The latter has to be taken also into 269 consideration when interpreting WBE results. For instance, the high alcohol consumption 270 figures obtained by Chen et al. in one of the communities investigated in the US between

271 June and September reflected the impact of tourist influx rather than increased consumption 272 of alcohol by residents living in the area [20]. One major limitation of WBE is that, 273 contrary to traditional methods, it does not provide alcohol consumption estimates for 274 specific population groups, and thus, consumption patterns by age or by gender cannot be 275 segregated.

\section{Conclusions}

278 The WBE approach is a very useful tool, complementary to traditional methods, to estimate 279 the consumption of alcohol in a population. It allows obtaining timely estimates, in a fast, 280 inexpensive, objective, and anonymous way. The uncertainty of this methodology can be 
281 reduced by selecting the best method to estimate population size and increasing the 282 knowledge on EtS stability in the catchment and alcohol metabolism. Data comparability 283 with WHO annual estimates for the different countries could be also improved by using 284 longer sampling periods and covering larger populations so that estimates obtained are 285 representative of a whole country and a year period.

286 With no doubt, the WBE approach is an already consolidated and valid tool to evaluate 287 changes in alcohol consumption patterns and the effectivity of control and preventive 288 measures applied in specific locations to reduce alcohol consumption.

289

290 Conflict of interest statement

291 Nothing to declare

292

293 Acknowledgments

294 This work has been financially supported by the Government of Catalonia (Consolidated 295 Research Group 2017 SGR 01404-Water and Soil Quality Unit).

296

297

298 
Table 1. Alcohol consumption estimates by means of the WBE approach

\begin{tabular}{|c|c|c|c|c|c|c|c|}
\hline \multirow[t]{2}{*}{ Level } & \multirow[t]{2}{*}{ City or country } & \multicolumn{2}{|c|}{$\begin{array}{c}\text { Alcohol consumption } \\
\text { (mL/day/inh.) }\end{array}$} & \multirow{2}{*}{$\begin{array}{l}\text { Normalized } \\
\text { population }\end{array}$} & \multirow[t]{2}{*}{ Period of time } & \multirow{2}{*}{$\begin{array}{l}\text { Exc. rate } \\
(\%) \text { used }\end{array}$} & \multirow[t]{2}{*}{ Ref. } \\
\hline & & Average & Range & & & & \\
\hline \multirow[t]{23}{*}{ Local } & Oslo (NO) & 16.1 & & Total population & 2009 (3 weeks) & $0.010-0.016$ & [8] \\
\hline & Santiago (ES) & 13.6 & $3.8-22.6$ & Total population & 2012-2013-2014 (one week) & 0.012 & {$[7,17]$} \\
\hline & Barcelona (ES) & 18 & $11-25$ & $>15$ years & 2013-2014-2015 (one week) & 0.011 & {$[14,15]$} \\
\hline & Milan (IT) & 5.1 & $3.2-10.5$ & Total population & 2012-2013-2014 (two weeks) & 0.012 & {$[17]$} \\
\hline & Valencia (ES) & $4.3-17.0$ & $1.1-56.1$ & $>15$ years & 2014 (17 days in 3 WWTPs) & $0.010-0.016$ & [9] \\
\hline & Normal week & $3.3-6.2$ & $1.1-18.31$ & & & & \\
\hline & Festivity & $6.4-42.7$ & $4.4-56.11$ & & & & \\
\hline & Canberra (AU) & 14.6 & $9.3-22.3$ & Total population & 2014 (one week) & 0.012 & {$[18]$} \\
\hline & Toowoomba (AU) & 9.7 & $6.9-14.5$ & & & & \\
\hline & Montreal (CA) & 29.2 & $21.8-38.8$ & & & & \\
\hline & Granby (CA) & 44.3 & $27.3-59.3$ & & & & \\
\hline & Lugano $(\mathrm{CH})$ & 6.5 & $4.5-8.4$ & & & & \\
\hline & Dortmund (DE) & 23.6 & 18.1-34 & & & & \\
\hline & Dülmen (DE) & 20.3 & $5.5-40$ & & & & \\
\hline & Dresden (DE) & 29.4 & $15.1-91.7$ & & & & \\
\hline & Munich (DE) & 29.5 & $0.5-47.4$ & & & & \\
\hline & Berlin (DE) & 16.9 & $13.8-22.3$ & & & & \\
\hline & Copenhagen (DK) & 40.2 & $24.6-74$ & & & & \\
\hline & Barcelona (ES) & 11.7 & $5.7-17.6$ & & & & \\
\hline & Castellón (ES) & 23.4 & $11.6-61.6$ & & & & \\
\hline & Milan (IT) & 6.4 & $5.1-8.1$ & & & & \\
\hline & Amsterdam (NL) & 22 & $14.3-30.5$ & & & & \\
\hline & Eindhoven (NL) & 21.7 & $13.7-30.4$ & & & & \\
\hline
\end{tabular}


Table 1 (cont.). Alcohol consumption estimates by means of the WBE approach

\begin{tabular}{|c|c|c|c|c|c|c|c|}
\hline \multirow[t]{2}{*}{ Level } & \multirow[t]{2}{*}{ City or country } & \multicolumn{2}{|c|}{$\begin{array}{l}\text { Alcohol consumption } \\
(\mathrm{mL} / \mathrm{day} / \mathrm{inh} .)\end{array}$} & \multirow{2}{*}{$\begin{array}{l}\text { Normalized } \\
\text { population }\end{array}$} & \multirow[t]{2}{*}{ Period of time } & \multirow{2}{*}{$\begin{array}{l}\text { Exc. rate } \\
(\%) \text { used }\end{array}$} & \multirow[t]{2}{*}{ Ref. } \\
\hline & & Average & Range & & & & \\
\hline & Utrecht (NL) & 12.9 & $7.7-20.7$ & Total population & 2014 (one week) & 0.012 & {$[18]$} \\
\hline & Oslo (NO) & 19.2 & $8.8-52.9$ & & & & \\
\hline & Almada (PT) & 14.6 & $8.4-24.1$ & & & & \\
\hline & London (UK) & 21.5 & $10.9-36$ & & & & \\
\hline & Brussels (BE) & 21.6 & & Total population & 2015 (one week) & 0.012 & {$[10]$} \\
\hline & Zurich $(\mathrm{CH})$ & 14.7 & & & & & \\
\hline & Copenhagen (DK) & 29.7 & & & & & \\
\hline & Castellón (ES) & 6.6 & & & & & \\
\hline & Milan (IT) & 6.6 & & & & & \\
\hline & Utrecht (NL) & 10.8 & & & & & \\
\hline & Oslo (NO) & 18.9 & & & & & \\
\hline & Bristol (UK) & 16.2 & & & & & \\
\hline & Lier (BE) & & & Total population & 2014 (four two-week periods) & 0.012 & [19] \\
\hline & Lesvos (GR) & $5.4 / 3.4$ & $2.2-11.2 / 1.7-7.2$ & Total population & 2015 (one week in 2 WWTPs) & 0.012 & [12] \\
\hline & Ho Chi Minh (Vietnam) & $3.1-3.9$ & & Total population & 2015 (one week, grab samples) & 0.012 & [16] \\
\hline & Midwestern and & 37 & & $>15$ years & 2015-2016 (one weekday every month & 0.012 & {$[20]$} \\
\hline & Northeastern region (U.S) & & & & for eleven months, 3 WWTPs) & & \\
\hline \multirow[t]{2}{*}{ National } & Belgium & & & $>15$ years & 2013-2015 (one week, 8 WWTPs) & 0.012 & [11] \\
\hline & Australia & $12-13$ & $7-24$ & $15-79$ years & 2014-2015 (one week, 18 WWTPs) & 0.012 & {$[13]$} \\
\hline
\end{tabular}




\section{References}

Papers of particular interest have been highlighted as:

* of special interest

** of outstanding interest

1. World Health Organization (WHO), 2018. Global status report on alcohol and health 2018. Available at https://goo.gl/kv8FD6. Accessed December 2018.

2. Moskalewicz J and Sieroslawski J, 2010. Drinking population surveys: guidance document for standardized approach. Warsaw: Institute of Psychiatry and Neurology. Available at: https://goo.gl/i15bws. Accessed on December 2018.

3. World Health Organization,2000. International guide for monitoring alcohol consumption and related harm. Geneva. Available at: https://qoo.ql/DbAjCp. Accessed on December 2018.

4. Sordo, L., G. Barrio, M.J. Bravo, J.R. Villalbí, A. Espelt, M. Neira, and E. Regidor, Estimating average alcohol consumption in the population using multiple sources: the case of Spain. Population health metrics, 2016. 14: p. 21-21.

5. Stockwell, T., J. Zhao, T. Greenfield, J. Li, M. Livingston, and Y. Meng, Estimating underand over-reporting of drinking in national surveys of alcohol consumption: identification of consistent biases across four English-speaking countries. Addiction (Abingdon, England), 2016. 111(7): p. 1203-1213.

6. Helander, A. and O. Beck, Ethyl sulfate: A Metabolite of Ethanol in Humans and a Potential Biomarker of Acute Alcohol Intake. Journal of Analytical Toxicology, 2005. 29.

7. Rodríguez-Álvarez, T., R. Rodil, R. Cela, and J.B. Quintana, lon-pair reversed-phase liquid chromatography-quadrupole-time-of-flight and triple-quadrupole-mass spectrometry determination of ethyl sulfate in wastewater for alcohol consumption tracing. Journal of Chromatography A, 2014. 1328: p. 35-42.

8. Reid, M.J., K.H. Langford, J. Mørland, and K.V. Thomas, Analysis and Interpretation of Specific Ethanol Metabolites, Ethyl Sulfate, and Ethyl Glucuronide in Sewage Effluent for the Quantitative Measurement of Regional Alcohol Consumption. Alcoholism: Clinical and Experimental Research, 2011. 35(9): p. 1593-1599.

9. Andrés-Costa, M.J., Ú. Escrivá, V. Andreu, and Y. Picó, Estimation of alcohol consumption during "Fallas" festivity in the wastewater of Valencia city (Spain) using ethyl sulfate as a biomarker. Science of The Total Environment, 2016. 541: p. 616-622.

10. Baz-Lomba, J.A., S. Salvatore, E. Gracia-Lor, R. Bade, S. Castiglioni, E. Castrignanò, A. Causanilles, F. Hernandez, B. Kasprzyk-Hordern, J. Kinyua, A.-K. McCall, A. van Nuijs, C. Ort, B.G. Plósz, P. Ramin, M. Reid, N.I. Rousis, Y. Ryu, P. de Voogt, J. Bramness, and K. Thomas, Comparison of pharmaceutical, illicit drug, alcohol, nicotine and caffeine levels in wastewater with sale, seizure and consumption data for 8 European cities. BMC Public Health, 2016. 16(1): p. 1035.

11. Boogaerts, T., A. Covaci, J. Kinyua, H. Neels, and A.L.N. van Nuijs, Spatial and temporal trends in alcohol consumption in Belgian cities: A wastewater-based approach. Drug and Alcohol Dependence, 2016. 160: p. 170-176. 
12. Gatidou, G., J. Kinyua, A.L.N. van Nuijs, E. Gracia-Lor, S. Castiglioni, A. Covaci, and A.S. Stasinakis, Drugs of abuse and alcohol consumption among different groups of population on the Greek Island of Lesvos through sewage-based epidemiology. Science of The Total Environment, 2016. 563-564: p. 633-640.

13. Lai, F.Y., C. Gartner, W. Hall, S. Carter, J. O'Brien, B.J. Tscharke, F. Been, C. Gerber, J. White, P. Thai, R. Bruno, J. Prichard, K.P. Kirkbride, and J.F. Mueller, Measuring spatial and temporal trends of nicotine and alcohol consumption in Australia using wastewater-based epidemiology. Addiction, 2018. 113(6): p. 1127-1136.

14. Mastroianni, N., M. Lopez de Alda, and D. Barcelo, Analysis of ethyl sulfate in raw wastewater for estimation of alcohol consumption and its correlation with drugs of abuse in the city of Barcelona. Journal of Chromatography A, 2014. 1360: p. 93-99.

15. Mastroianni, N., E. López-García, C. Postigo, D. Barceló, and M. López de Alda, Five-year monitoring of 19 illicit and legal substances of abuse at the inlet of a wastewater treatment plant in Barcelona (NE Spain) and estimation of drug consumption patterns and trends. Science of The Total Environment, 2017. 609: p. 916-926.

16. Nguyen, H.T., P.K. Thai, S.L. Kaserzon, J.W. O'Brien, G. Eaglesham, and J.F. Mueller, Assessment of drugs and personal care products biomarkers in the influent and effluent of two wastewater treatment plants in Ho Chi Minh City, Vietnam. Science of The Total Environment, 2018. 631-632: p. 469-475.

17. Rodríguez-Álvarez, T., I. Racamonde, I. González-Mariño, A. Borsotti, R. Rodil, I. Rodríguez, E. Zuccato, J.B. Quintana, and S. Castiglioni, Alcohol and cocaine co-consumption in two European cities assessed by wastewater analysis. Science of The Total Environment, 2015. 536: p. 91-98.

18. Ryu, Y., D. Barceló, L.P. Barron, L. Bijlsma, S. Castiglioni, P. de Voogt, E. Emke, F. Hernández, F.Y. Lai, A. Lopes, M. López de Alda, N. Mastroianni, K. Munro, J. O'Brien, C. Ort, B.G. Plósz, M.J. Reid, V. Yargeau, and K.V. Thomas, Comparative measurement and quantitative risk assessment of alcohol consumption through wastewater-based epidemiology: An international study in 20 cities. Science of The Total Environment, 2016. 565: p. 977-983.

19. van Wel, J.H., E. Gracia-Lor, A.L. van Nuijs, J. Kinyua, S. Salvatore, S. Castiglioni, J.G. Bramness, A. Covaci, and G. van Hal, Investigation of agreement between wastewaterbased epidemiology and survey data on alcohol and nicotine use in a community. Drug Alcohol Depend, 2016. 162.

20. Chen, J., A.K. Venkatesan, and R.U. Halden, Alcohol and nicotine consumption trends in three U.S. communities determined by wastewater-based epidemiology. Science of The Total Environment, 2019. 656: p. 174-183.

21. Castiglioni, S., L. Bijlsma, A. Covaci, E. Emke, F. Hernández, M. Reid, C. Ort, K.V. Thomas, A.L.N. van Nuijs, P. de Voogt, and E. Zuccato, Evaluation of Uncertainties Associated with the Determination of Community Drug Use through the Measurement of Sewage Drug Biomarkers. Environmental Science \& Technology, 2013. 47(3): p. 1452-1460.

22. Gao, J., J. Li, G. Jiang, Z. Yuan, G. Eaglesham, A. Covaci, J.F. Mueller, and P.K. Thai, Stability of alcohol and tobacco consumption biomarkers in a real rising main sewer. Water Research, 2018. 138: p. 19-26.

23. Banks, A.P.W., F.Y. Lai, J.F. Mueller, G. Jiang, S. Carter, and P.K. Thai, Potential impact of the sewer system on the applicability of alcohol and tobacco biomarkers in wastewaterbased epidemiology. Drug Testing and Analysis, 2018. 10(3): p. 530-538. 
24. Høiseth, G., J.P. Bernard, N. Stephanson, P.T. Normann, A.S. Christophersen, J. Mørland, and $A$. Helander, Comparison between the urinary alcohol markers EtG, EtS, and GTOL/5HIAA in a controlled drinking experiment. Alcohol and Alcoholism, 2008. 43(2): p. 187-191.

25. Wurst, F.M., S. Dresen, J.P. Allen, G. Wiesbeck, M. Graf, and W. Weinmann, Ethyl sulphate: a direct ethanol metabolite reflecting recent alcohol consumption. Addiction, 2006. 101(2): p. 204-211.

26. Chen, C., C. Kostakis, J.P. Gerber, B.J. Tscharke, R.J. Irvine, and J.M. White, Towards finding a population biomarker for wastewater epidemiology studies. Science of The Total Environment, 2014. 487: p. 621-628.

27. Lai, F.Y., C. Ort, C. Gartner, S. Carter, J. Prichard, P. Kirkbride, R. Bruno, W. Hall, G. Eaglesham, and J.F. Mueller, Refining the estimation of illicit drug consumptions from wastewater analysis: co-analysis of prescription pharmaceuticals and uncertainty assessment Water Research, 2011. 45: p. 4437-4448.

28. Been, F., L. Rossi, C. Ort, S. Rudaz, O. Delemont, and P. Esseiva, Population normalization with ammonium in wastewater-based epidemiology: application to illicit drug monitoring. Environmental Science \& Technology, 2014. 48: p. 8162-8169.

29. Thomas, K.V., A. Amador, J.A. Baz-Lomba, and M. Reid, Use of Mobile Device Data To Better Estimate Dynamic Population Size for Wastewater-Based Epidemiology. Environmental Science \& Technology, 2017. 51(19): p. 11363-11370. 


\section{References}

Papers of particular interest have been highlighted as:

* of special interest

** of outstanding interest

8 - This is the first study that uses EtS concentrations in water to estimate alcohol consumption at the community level. $\left({ }^{*}\right)$

9 - This study shows that the use of WBE allows detecting changes in alcohol consumption caused by a local festivity. $(*)$

13 - This is the first study that applies the WBE approach at the national level with very large population coverage ( $43 \%$ of total population). $\left({ }^{* *}\right)$

21 - Authors evaluate and quantitate the different sources of uncertainty associated with consumption estimates derived from the wastewater based epidemiology, and provide best practice protocols. $(* *)$

28 - This study evaluates the use of ammonium level in wastewater as a marker of fluctuations in population size

29 - This study shows how population estimates may affect WBE-estimates and how population size can be accounted for using mobile devices data. $(*)$ 